\title{
Recurrent anaphylaxis in patient allergic to eggplant - a case study
}

\author{
Natalia Ukleja-Sokołowska, ${ }^{1}$ Ewa Gawrońska-Ukleja, ${ }^{1}$ Magdalena Żbikowska-Gotz, ${ }^{1}$ Lukasz Sokołowski, ${ }^{2}$ Zbigniew Bartuzi ${ }^{1}$
}

\begin{abstract}
Eggplant allergy is rare and most of the previously described reactions were mild. In this case report, we present an interesting case of a 27-year-old male who experienced symptoms of anaphylaxis (shortness of breath, and swelling of the face, lips and tongue, which was followed by hypotension, tachycardia of 140/min and a sudden loss of consciousness) several minutes after eating a dish containing backed eggplant. Previously, the patient had experienced symptoms of allergy after eating different types of food, such as salad and chicken in spices. The symptoms were enhanced by co-factors. They were heterogeneous, ranging from oral symptoms to anaphylactic shock.

During the diagnostic pathway, skin prick tests (SPTs) were positive to grass and cat. Prick by prick skin tests were positive for eggplant, both cooked and fresh. In ImmunoCap ISAC, IgE specific to rPhl p 1 (1.7 ISU-E), rCan f 5 (1,2 ISU-E), Fel d 1 (9.6 ISU-E ) and LTPs - nJug r 3 (0.5 ISU-E), rPru p 3 (0.6 ISU-E), rPla a 3 (1.3 ISU-E) were found.

Based on the clinical pattern of the disease and the results of component resolved diagnosis, we suspect that this complex case of anaphylaxis may be gathered with LTP syndrome.
\end{abstract}

Keywords: anaphylaxis, eggplant, allergy, component resolved diagnosis, lipid transfer protein

\section{From:}

${ }^{1}$ Department and Clinic of Allergology, Clinical Immunology and Internal Diseases, Ludwik Rydygier Collegium Medicum in Bydgoszcz NCU, Poland

${ }^{2}$ Department of Hygiene, Epidemiology and Ergonomics, Division of Ergonomics and Exercise Physiology, Ludwik Rydygier Collegium Medicum in Bydgoszcz NCU, Poland

\section{Introduction}

Allergic reactions to eggplant (aubergine, Solanum melongena) have been rarely reported and are mainly attributed to cross-reactivity with proteins of other fruit and vegetables. ${ }^{1}$

It is widely known that the frequency of allergy to different types of allergens depends on a number of factors, including exposure to a given allergen, socioeconomic factors, comorbidities, the climate zone, and dietary habits., ${ }^{2,3}$ The currently described patient is probably one of the first in Poland who has experienced anaphylactic reaction due to eggplant allergy.

The prevalence of IgE-mediated eggplant allergy is estimated at approximately $0.8 \%$, with higher rates of sensitization in females. ${ }^{4}$ Eggplant is generally eaten after cooking or baking. ${ }^{5}$ Eggplant contains 48 proteins that con potentially be a source of allergic reaction, most of which are not yet fully described. ${ }^{6}$ One of the most interesting known eggplant allergens is LTP (lipid transfer protein), which is potentially cross-reactive with allergens of other fruit and vegetables. Other allergens include a protein with weak homology to $7 \mathrm{~S}$ vicilins, ${ }^{7}$ lipoxygenase, protein proteinase

\section{Corresponding author:}

Natalia Ukleja-Sokołowska

ul. Ujejskiego 75

85-168 Bydgoszcz

E-mail: ukleja@10g.pl

inhibitor and $15 \mathrm{kDa}$ and $35 \mathrm{kDa}$ heat stable proteins with unknown functions. ${ }^{8}$

In 2009, a very interesting work was published by Babu et al., in which the authors investigated sensitization to different parts of the eggplant fruit, and detected the allergens. It was noticed that most allergens were localized in the eggplant peel (9 allergens; $26-71 \mathrm{kDa}$ range) rather than the pulp (3 allergens; $52-71 \mathrm{kDa}$ ); among these, the allergens with molecular weights of $26,28,36$, and $71 \mathrm{kDa}$ seemed to be heat-stable, and the authors detected the allergens with molecular weights of 43, 45, 64 , and $71 \mathrm{kDa}$ to be glycoproteins. ${ }^{5}$

Lipid transfer proteins (LTPs) are plant panallergens, which are described as pure food allergens, and are capable of sensitizing through the gastrointestinal tract. LTPs have been found in many plant-related foods, particularly in those belonging to the Rosaceae family, for example peach (Pru $\mathrm{p}$ 3) and apple ( Mal d 3), but also hazelnut (Cor a 8) from the Betulaceae family, walnut (Jug r 3) from the Juglandaceae family, peanut (a legume crop, from the Fabaceae family), sesame (Pedaliaceae family), and celery (Apiaceae family)., ${ }^{9,10}$ 
nsLTPs (non-specific LTPs) are mostly present in the peel of fruits, rather than the pulp. ${ }^{11,12}$ Interestingly, symptoms after the consumption of LTP-containing foods can differ. In 2006, Sancho et al. showed that nsLTP levels in apple are greatly dependent on maturity, storage conditions and cultivar. ${ }^{13}$

LTP syndrome is a relatively new name for a clinical pattern of symptoms and natural course of allergy to this panallergen. We now know that there are different recognition patterns of LTPs - some patients only react to peach and apple (Rosaceae fruit, where sensitization to peach LTP Pru p 3 can be a marker), while others react to a wide range of foods, including hazelnut or walnut (were mugwort Art v 3 can be a marker). ${ }^{14,15}$ Primary sensitization may result from exposure through the oral route, although skin contact may also lead to allergic reactions. This type of allergy is common in the Mediterranean, but not unheard of in other parts of the world. Peach is a typical primary sensitizer..$^{10}$ The symptoms may vary from mild oral allergy syndrome (OAS) to life-threatening anaphylactic reaction, even in a single patient. ${ }^{9,11}$ The concentration of LTP differs in fruits and vegetables and increases with storage conditions and in certain cultivars. ${ }^{13}$

\section{A case study}

Here, we present the case of a 27-year-old male admitted to the hospital with recurrent anaphylactic reactions of unknown cause.

The first allergic reaction appeared in summer 2012; the patient ate a seafood salad with a mixture of vegetables and then went for a fast walk, during which time he experienced swelling of the nose, cheeks, eyelids and tongue, tearing, rhinorrhea and tachycardia. The symptoms retreated after the administration of steroids and antihistamines.

On $31^{\text {st }}$ October 2013, the patient ate fried chicken in a mixture of spices, with a tomato and cucumber salad. Immediately after ingestion he suffered an incident of dyspnea, with swelling of the face and severe pruritic urticaria on his back and upper limbs. In the emergency room, he received steroids and antihistamines with good clinical effect.

On $14^{\text {th }}$ October 2014, the patient ate a homemade dish with pork and eggplant, and drank one beer. Initially, he experienced shortness of breath, and swelling of the face, lips and tongue, which was followed by hypotension, tachycardia of $140 / \mathrm{min}$ and a sudden loss of consciousness. He was given epinephrine, crystalloid fluids, steroids and antihistamines, was hospitalized and then discharged with a recommendation to use adrenaline in an auto-injector in the case of anaphylactic reaction.

During the diagnostic pathway, skin prick tests (SPTs) were performed with common inhalant and food allergens (Allergopharma, Nexter); also, prick by prick skin tests were undertaken with selected foods (raw and cocked eggplant pulp and peel, shrimp, banana, scallops, crab, beer, lettuce, tomato, cucumber, chicken meat, pork). The concentrations of IgE against selected food allergens was measured using the ImmunoCap system and the levels of IgE specific to allergen components was established using ImmunoCap ISAC; a value $>=0.35 \mathrm{kU} / \mathrm{l}$ was considered positive, in accordance with practice commonly accepted in other studies. ${ }^{16}$
The patient had a positive (interpretation according to The Global Allergy and Asthma European Network (GA'LEN)) ${ }^{17}$ SPT to allergens of grass and cat dander, but negative results for birch, alder, hazel, weeds, dust mites, molds, and dog dander. Food SPTs were positive to peach, but negative to apple, orange, strawberry, banana, carrot, tomato, celery, potato, hazelnut, peanut, wheat flour, rye flour, rice, soybean, egg, cod, cocoa, and pork.

Results of the prick by prick tests with fresh and cooked eggplant were positive - raw eggplant peel $30 / 30 \mathrm{~mm}$, pulp $10 / 10$ $\mathrm{mm}$, cooked eggplant peel $7 / 8 \mathrm{~mm}$, pulp $4 / 4 \mathrm{~mm}$ (histamine $4 / 4 \mathrm{~mm}$, control $0 / 0 \mathrm{~mm}$ ) and negative with other tested native allergens. Specific IgE concentration was elevated against grass mix (Orchard-, Meadow Fescue, Perennial Rye-, Timothy-, Bluegrass) $-1.63 \mathrm{IU} / \mathrm{ml}$ and cat $-0.96 \mathrm{IU} / \mathrm{ml}$. In Poland, it is not possible to estimate IgE to eggplant due to a lack of allergen extract.

The results of ImmunoCap ISAC are displayed in Table $\mathbf{1 .}$ In ImmunoCap ISAC, there are no allergen components of eggplant available.

Table 1. ImmunoCap ISAC results, only positive results acknowledged

\begin{tabular}{lll}
\hline Allergen component & Level of asIgE (ISU-E) & Allergen source \\
\hline rPhl p 1 & 1.7 & Timothy \\
\hline rCan f 5 & 1.2 & Dog \\
\hline Fel d 1 & 9.6 & Cat \\
nJug r 3 & 0.5 & Walnut (LTP) \\
\hline rPru p 3 & 0.6 & Peach (LTP) \\
rPla a 3 & 1.3 & Plane-tree (LTP) \\
\hline
\end{tabular}

Negative results to following allergen components: $\mathrm{nGal} \mathrm{d} \mathrm{1,} \mathrm{nGal} \mathrm{d} \mathrm{2,} \mathrm{nGal} \mathrm{d}$ 3, nGal d 5, nBos d 4, nBos d 5, nBos d 6, nBos d 8, nBos d lactoferrin, rGad c 1, nPen $\mathrm{m} 1$, nPen $\mathrm{m}$ 2, nPen $\mathrm{m} 4$, rAna o 2 , rBer e 1, rCor a 1.0401, rCor a 8, nCor a 9, nJug r 1, nJug r 2, nSes i 1, rAra h 1, rAra h 2, rAra h 3, nAra h 6, rAra h 8, rAra h 9, rGly m 4, nGly m 5, nGly m 6, nFag e 2, rTri a 14, rTri a 19.0101, nTri a aA_TI, nAct d 1, nAct d 2, nAct d 5, rAct d 8, rApi g 1, rMal d 1, rPru p 1, nCyn d 1, rPhl p 2, nPhl p 4, rPhl p 5, rPhl p 6, rPhl p 7, rPhl p 11, rPhl p 12, rAln g 1, rBet v 1, rBet v 2, rBet v 4, rCor a 1.0101, nCry j 1, nCup a 1, nOle e 1, nOle e 7 , rOle e 9 , rPla a 1, nPla a 2, nAmb a 1, nArt v 1, nArt v 3 , rChe a 1 , rMer a 1 , rPar j 2, rPla 1 1, nSal k 1, rCan f 1, rCan f 2, nCan f 3, rEqu c 1, nEqu c 3, nFel d 2, rFel d 4, nMus m 1, rAlt a 1, rAlt a 6, rAsp f 1, rAsp f 3, rAsp f 6, rCla h 8, rBlo t 5, nDer f 1, rDer f 2, nDer p 1, rDer p 2, rDer p 10, rLep d 2, rBla g 1, rBla g 2, rBla g 5, nBla g 7, rApi m 1, nApi m 4, rPol d 5, rVes v 5, rAni s 1, rAni s 3, rHev b 1, rHev b 3, rHev b 5, rHev b 6.01, rHev b 8, nMUXF3

\section{Discussion}

Based on the medical interview and the results of in vitro and in vivo tests, we established that the patient is strongly allergic to eggplant, grass and cat dander, although it does not explain all of the symptoms. The fourth anaphylactic reaction was clear, with anaphylaxis due to eggplant consumption. All other reactions were related to different kinds of food, in particularl vegetables.

The clinical pattern of the disease and low, but still present levels of asIgE (antigen specific immunoglobulin E) directed against LTPs from different plant sources may suggest that this 
patient suffers from LTP syndrome. It is worth emphasizing the features of this case typical for LTP syndrome - unstable clinical course, role of co-factors (exercise, alcohol) and recurrent symptoms after the consumption of fruits and vegetables from different sources.

There may be several reasons for low levels of LTPs in our patient. An important factor is the time between exposure to LTPs and an examination of the patients' blood serum. In 2011, Asero et al. found that specific IgE levels are only partially predictive of clinical allergy in LTP allergic patients. ${ }^{18}$

Similar results were published in 2012 by Pascal et al., who also found that there was no correlation between LTP-specific IgE levels and the severity of symptoms. In their research, the main offending foods reported by LTP allergic patients and confirmed by SPTs were peach, lettuce, walnut, hazelnut, peanut and green bean. In $40 \%$ of patients, cofactors were necessary to induce symptoms. ${ }^{19}$ In the present case, cofactors (exercise and alcohol) played an important role in the development of anaphylaxis.

The pathogenic mechanism of cofactors in food allergy is still unclear. An interesting theory suggests that changes in mucosal permeability induced by exercise, medication or other cofactors can enhance allergen absorption through the mucous membranes, and, as a result, enhances exposure to allergens. ${ }^{20}$

Cases of cross allergy between eggplants and latex allergens were described. ${ }^{21}$ In the case of our patient, there were no symptoms related to latex and skin prick tests with latex allergen extracts were negative.

The management of patients allergic to LTP is very important, due to large number of anaphylactic reactions in patients with this type of allergy. In 2014, it was emphasized that in the apple allergic population, LTP is most often involved in the occurrence of generalized symptoms in patients. $^{22}$ The current patient was advised to carry adrenalin in an auto-injector after his $3^{\text {rd }}$ emergency room visit. It is unfortunately not surprising; there have been researches which state that the majority of patients with anaphylaxis do not receive self-injectable adrenaline to prevent future anaphylactic shock. $^{23}$

Avoidance of sensitizing products is a key part in the management of food allergy. Due to cross-reactivity of nsLTPs, symptoms may appear after the ingestion of fruit and vegetables which previously seemed safe. Patients at risk of anaphylaxis, should carry a rescue set, including adrenaline in an auto-injector. Some patients that are allergic to the LTPs of Rosaceae fruits (around 1/3) may tolerate the pulp of these fruits. Unfortunately, reactions can still be observed when a cofactor is associated. Therefore, as a general recommendation, patients should avoid peeled fruits as well. ${ }^{19}$

In 2007, Asero et al. searched for safe plant-derived foods for LTP-allergic patients. He came to the conclusion that carrot, potato, banana and melon seem safe. ${ }^{24}$

The future for LTP syndrome patients definitely involves a specific immunotherapy developed according to the patient -specific sensitivity profile. There are some interesting cases showing the efficiency of this treatment, but there are no commercially available immunotherapies for these patients. ${ }^{25}$
Knowledge of the clinical pattern of allergy may help patients to understand their disease and prevent life-threatening anaphylactic reactions.

\section{Conclusion}

The described patient suffers from a life threatening food allergy. Eggplant allergy, which is very rare in Europe, was confirmed by prick by prick tests. The patient was advised to carry adrenaline in an auto-injector, as well as steroids and antihistamines in case of an anaphylactic reaction.

The clinical pattern of the disease and low, but still present levels of asIgE directed against LTPs from different plant sources, may suggest that this patient suffers from LTP syndrome.

\section{List of abbreviations}

Lipid transfer proteins - LTPs

nsLTP - non-specific LTS

Skin prick test - SPT

Oral allergy syndrome - OAS

Antigen specific immunoglobulin E - asIgE

Component resolved diagnosis - CRD

\section{Competing Interests}

The authors declare that they have no competing interests.

\section{Financial support}

No financial source to declare.

\section{Authors' Contributions}

Natalia Ukleja-Sokołowska - interviewed the patient, performed skin prick tests with native allergens, obtained patient permission and drafted the manuscript.

Ewa Gawrońska-Ukleja - designed and coordinated the study, and reviewed the manuscript

Magdalena Żbikowska-Gotz - performed the immunoassays.

Łukasz Sokołowski - reviewed the manuscript, drafted necessary corrections

Zbigniew Bartuzi - reviewed the manuscript, study design and patient diagnosis.

All authors read and approved the final manuscript.

\section{References}

1. Pramod SN, Venkatesh YP. Allergy to eggplant (Solanum melongena). J Allergy Clin Immunol. 2004;113:171-3.

2. Tham EH, Lee AJ., Bever HV., Aeroallergen sensitization and allergic disease phenotypes in Asia, Asian Pac J Allergy Immunol. 2016 Sep;34(3): 181-189.

3. An SY, Choi HG, Kim SW, Park B, Lee JS, Jang JH, Sung MW., Analysis of various risk factors predisposing subjects to allergic rhinitis., Asian Pac J Allergy Immunol. 2015 Jun;33(2):143-51.

4. Harish Babu BN, Mahesh PA, Venkatesh YP. A cross-sectional study on the prevalence of food allergy to eggplant (Solanum melongena L.) reveals female predominance. Clin Exp Allergy. 2008;38:1795-802

5. Babu BN, Venkatesh YP. Clinico-Immunological Analysis of Eggplant (Solanum melongena) Allergy Indicates Preponderance of Allergens in the Peel. World Allergy Organ J. 2009;2:192-200 
6. Ramesh KR, Hemalatha R, Vijayendra CA, Arshi UZ, Dushyant SB, Dinesh KB., Transcriptome analysis of Solanum melongena L. (eggplant) fruit to identify putative allergens and their epitopes., Gene. 2016 Jan 15;576(1 Pt 1):64-71

7. Jain A, Salunke DM., Purification, identification and preliminary crystallographic studies of an allergenic protein from Solanum melongena., Acta Crystallogr F Struct Biol Commun. 2015 Feb;71(Pt 2):221-5

8. Hoseini-Alfatemi SM, Bayry J, Sharifi-Rad J., IgE response to two new allergen proteins of Solanum melongena L. (eggplant)., Immunol Lett. 2015 Dec;168(2):268-70

9. Pastorello EA, Robino AM. Clinical role of lipid transfer proteins in food allergy. Mol Nutr Food Res. 2004;48:356-62

10. Asero R., In patients with LTP syndrome food-specific IgE show a predictable hierarchical order., Eur Ann Allergy Clin Immunol. 2014 Jul;46(4):142-6.

11. Zuidmeer L, van Ree R. Lipid transfer protein allergy: primary food allergy or pollen/food syndrome in some cases. Curr Opin Allergy Clin Immunol. 2007;7:269-73.

12. Marzban G, Puehringer H, Dey R, Brynda S, Ma Y, Martinelli A, et al. Localization and distribution of the major allergens in apple fruits. Plant Sci. 2005; 169:387-394.

13. Sancho AI, Foxall R, Rigby NM, Browne T, Zuidmeer L, van Ree R, et al. Maturity and storage influence on the apple (Malus domestica) allergen Mal d 3, a nonspecific lipid transfer protein. J Agric Food Chem. 2006;54: 5098-104.

14. García-Sellés FJ, Díaz-Perales A, Sánchez-Monge R, Alcántara M, Lombardero M, Barber D, et al. Patterns of reactivity to lipid transfer proteins of plant foods and Artemisia pollen: an in vivo study. Int Arch Allergy Immunol. 2002:128:115-22.

15. Fernández-Rivas M, Benito C, González-Mancebo E, de Durana DA, Allergies to fruits and vegetables. Pediatr Allergy Immunol. 2008;19:67581 .
16. Ukleja-Sokolowska N, Gawronska-Ukleja E, Zbikowska-Gotz M, Socha E, Lis K, Sokołowski L, et al. Analysis of feline and canine allergen components in patients sensitized to pets., Allergy Asthma Clin Immunol. 2016 Nov 30;12:61.

17. Heinzerling L, Mari A, Bergmann KC, Bresciani M, Burbach G, Darsow U, et al., The skin prick test - European standards., Clin Transl Allergy. 2013 Feb 1;3(1):3

18. Asero R, Arena A, Cecchi L, Conte ME, Crivellaro M, Emiliani F, et al. Are IgE levels to foods other than rosaceae predictive of allergy in lipid transfer protein-hypersensitive patients? Int Arch Allergy Immunol. 2011;155:149-54.

19. Pascal M, Muñoz-Cano R, Reina Z, Palacín A, Vilella R, Picado C, et al. Lipid transfer protein syndrome: clinical pattern, cofactor effect and profile of molecular sensitization to plant-foods and pollens. Clin Exp Allergy. 2012;42:1529-39

20. Lambert GP, Boylan M, Laventure JP, Bull A, Lanspa S. Effect of aspirin and ibuprofen on GI permeability during exercise. Int J Sports Med. 2007;28:722-6.

21. Lee J, Cho YS, Park SY, Lee CK, Yoo B, Moon HB, et al. Eggplant anaphylaxis in a patient with latex allergy. J Allergy Clin Immunol. 2004;113:995-6.

22. Gomez F, Aranda A, Campo P, Diaz-Perales A, Blanca-Lopez N, Perkins J, et al. High prevalence of lipid transfer protein sensitization in apple allergic patients with systemic symptoms. PLoS One. 2014;9:e107304

23. Braganza S, Acworth J, Mckinnon D, Paediatric emergency departmen anaphylaxis: different patterns from adults. Arch Dis Child. 2006;91: 159-63.

24. Asero R, Mistrello G, Roncarolo D, Amato S, Detection of some safe plant-derived foods for LTP-allergic patients. Int Arch Allergy Immunol. 2007;144:57-63.

25. Pereira C, Bartolomé B, Asturias JA, Ibarrola I, Tavares B, Loureiro G, et al. Specific sublingual immunotherapy with peach LTP (Pru p 3). One year treatment: a case report., Cases J. 2009;2:6553. 